\title{
Unanticipated difficult airway due to undiagnosed oropharyngeal stenosis: a case report
}

\author{
Mina Nishimori ${ }^{1 *}$, Miyako Matsumoto ${ }^{2}$, Hideki Nakagawa $^{3}$ and Noriko Ichiishi ${ }^{1}$
}

\begin{abstract}
Unanticipated difficult airway is a challenging problem for anesthesiologists. Oropharyngeal stenosis (OPS) is a rare complication of upper airway surgery which may cause difficult airway. We present a patient whose postsurgical OPS was revealed during the induction of general anesthesia, and necessitated reschedule of surgery and tracheotomy. We also discuss the etiology and risk factors of postsurgical OPS.
\end{abstract}

Keywords: Oropharyngeal stenosis, Difficult airway, Upper airway surgery

\section{Background}

An unexpected difficult airway during the induction of general anesthesia is a condition that is best avoided. A difficult intubation can occur because of anatomical abnormalities or situational factors such as airway inflammation. Anatomical factors indicative of difficult airway include high body mass index, older age, Mallampati grade III or IV, severely limited jaw protrusion, and thyromental distance of less than $6 \mathrm{~cm}$ [1]. However, even those predictors could fail at predicting difficult laryngoscope. We present a case of unexpected difficult intubation due to undiagnosed oropharyngeal stenosis (OPS).

\section{Case presentation}

A 46-year-old $48 \mathrm{~kg}, 158 \mathrm{~cm}$ Chinese woman was scheduled for elective colectomy. Because she did not speak Japanese or English, we used a medical history questionnaire written in Chinese. She did not indicate any condition or surgical history. Although an interpreter (a Japanese nurse who spoke some Chinese) helped us with the preanesthesia visit, a detailed interview was unable. She was classified as Mallampati IV (uvula not visible), but we did not find other factors indicative of a difficult airway (BMI $<25$, interincisal distance $>6 \mathrm{~cm}$, thyromental distance $>6 \mathrm{~cm}$, jaw protrusion not limited, neck

\footnotetext{
*Correspondence: nishimori@seibokai.or.jp

${ }^{1}$ Department of Anesthesiology, Seibo International Catholic Hospital, 2-5-1,

Nakaochiai, Shinjuku, Tokyo 161-8521, Japan

Full list of author information is available at the end of the article
}

flexion-extension $>90$ degree). A rapid induction and tracheal intubation using a direct laryngoscope was scheduled, with a fiberscope made readily available.

General anesthesia was induced with fentanyl $100 \mathrm{mcg}$, propofol $100 \mathrm{mg}$ and rocuronium $40 \mathrm{mg}$. Mask ventilation was easy. Under direct laryngoscopy (Macintosh \#3), we could not observe the epiglottis because there was a membrane-like tissue in her pharynx with a narrow opening. When we examined her airway with a fiberscope, we could not observe the larynx through the opening, and we found the stenotic segment to be stiff and not expandable. We discussed the possibility of awakening the patient and performing a fibreoptic intubation because we believed that glossoptosis due to general anesthesia could have caused the larynx to be unobservable. However we decided to postpone the surgery for two reasons: firstly, the opening seemed to be too stiff and narrow for an endotracheal tube to pass through, and secondly, we had no information about the cause and etiology of her airway stenosis. Sugammadex $200 \mathrm{mg}$ was administered, and spontaneous respiration was resumed within five minutes. She came out of anesthesia without difficulty breathing and was sent back to the ward.

The next day, her airway was evaluated by an otolaryngologist. Laryngofiberscopy revealed an all-round fibrous cicatrix located between the tongue base and lateralposterior wall of the pharynx obliterating the oropharynx, including the uvula (Fig. 1). The diameter of the opening 


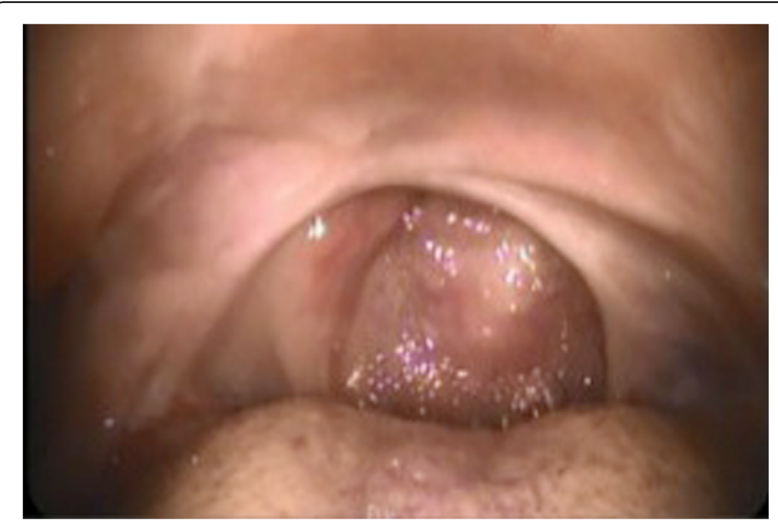

Fig. 1 Laryngofiberscopic view of the oropharynx. Adhesion of the anterior tonsillar pillars and inferior tonsillar fossa to the tongue base is observed. Uvula is obliterated

of the cicatrix was about $6 \mathrm{~mm}$ (Fig. 2). With the patient awake, we could observe the larynx through the opening and found the supraglottic space and the vestibule to be intact (Fig. 3). We also hired a professional medical interpreter (a Chinese native speaker) to record her medical history in detail. It revealed that she undertook adenotonsillectomy under local anesthesia at the age of 23 in China, and since then she had been suffering from dysphagia (difficulty swallowing solid food). She had lived on softened food and had adapted well to this problem. Since she did not consider this a serious problem and did not experience any other symptoms such as speech problems and difficulty breathing, she did not tell us about it on our preoperative visit.

It was possible to insert a $3 \mathrm{~mm}$ fiberscope through the opening of the cicatrix and through the vocal code; awake-fibreoptic intubation seemed to be possible. However, the difficulty of respiratory management using a tracheal tube of less than $6 \mathrm{~mm}$ of outer diameter (which means an inner diameter of 4 to $4.5 \mathrm{~mm}$ ) and

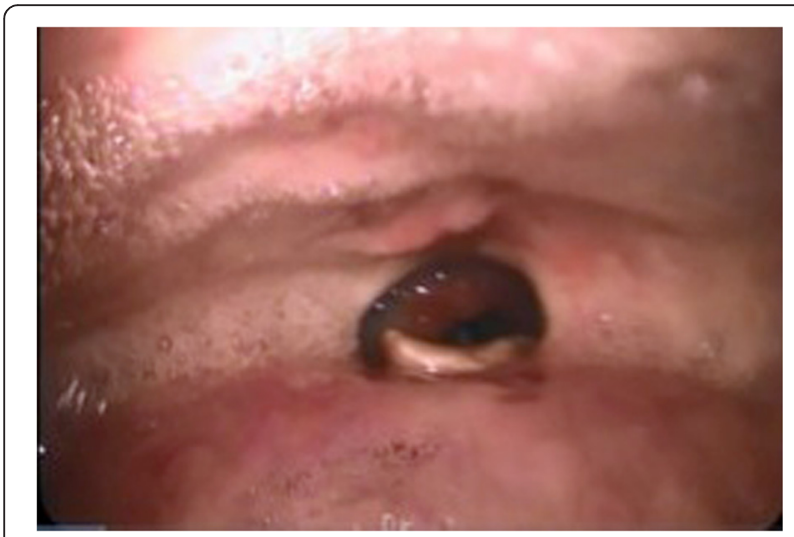

Fig. 2 Laryngofiberscopic view of the cicatrix. The diameter was about $6 \mathrm{~mm}$. Epiglottis is observed beyond the opening

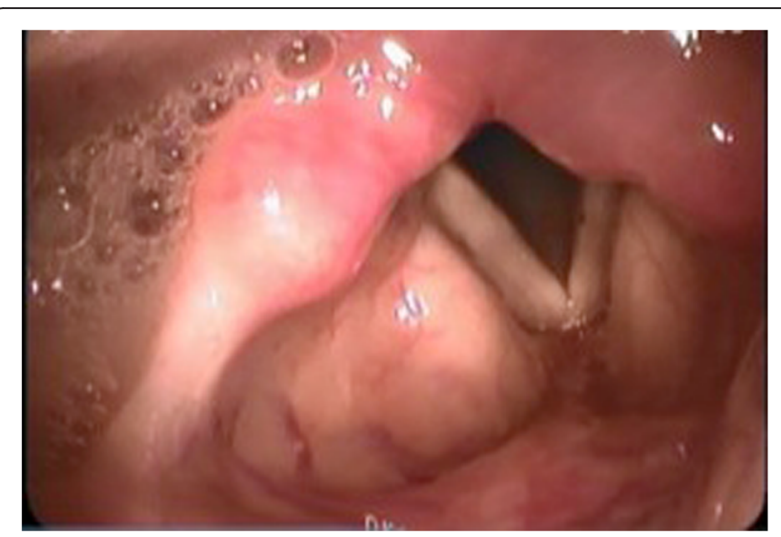

Fig. 3 Laryngofiberscopic view beyond the cicatrix. The supraglottic space and the vestibule are intact

the postoperative possible upper airway obstruction due to inflammation or scarring of the narrow opening, led to our decision to secure the airway by a tracheotomy. After the tracheotomy under local anesthesia, general anesthesia was induced and surgery was performed uneventfully.

\section{Discussion}

Oropharyngeal stenosis is caused by adhesion of the anterior pillars and inferior tonsillar fossa to the tongue base. Symptoms may include dyspnea on exertion, dysphagia, poor weight gain, and obstructive sleep apnea. Some patients may be asymptomatic [2]. Before 1940, most cases were due to infection, mainly pharyngeal syphilis [3]. After 1940, the most common etiology of OPS has been reported to be surgical trauma associated with adeno-tonsillectomy [2]. Risk factors for developing OPS after adeno-tonsillectomy include excessive cautery, postoperative bleeding and infection, and keloid tendency [2], but the prevalence has been reported to be very small [2]. This case was thought to be a rare case of OPS caused by adeno-tonsillectomy. Other causes include airway burn and systemic inflammatory diseases such as sarcoidosis [4] and Behçet's syndrome [5].

However, recent studies report higher prevalence of postoperative OPS among the patients who underwent more complicated upper-airway surgery such as pharyngoplasty for obstructive sleep apnea (OSA) [6, 7]. Prager et al. indicated the prevalence of postoperative OPS to be $8.2 \%$ in their retrospective chart review of 104 pediatric patients who underwent multilevel upper airway surgery including lingual tonsillectomy [8]. Cohen et al. reviewed their 65 adult patients who underwent surgery for OSA. Among the patients who underwent UPPP (uvulopalatopharyngoplasty) or TORS (trans-oral robotic surgery) including tongue-base resection, the prevalence of postoperative OPS was $7.8 \%$ [9]. In both reports, the authors indicate a single-stage procedure 
including tongue-base resection as a risk factor, along with the aforementioned risk factors of postoperative OPS $[8,9]$.

Several case series of post-surgical OPS are written by otolaryngologists $[2,3,8,9]$. One of them briefly commented on unexpected difficult airway during the induction of general anesthesia [2], but most of them did not. Although there is a case report of unanticipated difficult intubation due to an undiagnosed pharyngeal stenosis [10], this complication is still not widely recognized by anesthesiologists.

Our case raises two issues into discussion; the lack of preoperative interview and the awareness of postoperative OPS can cause unexpected difficult airway. For this particular case, there was a serious communication problem. Due to the language barrier, we were unable to obtain the information about the patient's history of adeno-tonsillectomy and subsequent dysphagia, because the patient considered it as a trivial problem. Ideally, medical interpreters should always be available, but this was not the case for our facility, as well as for many others. With the increase of patients unable to speak Japanese, this issue is becoming more common. In addition, even if we had no language issues and were aware of her surgical history, we could have overlooked her OPS because it is a very rare complication of adeno-tonsillectomy. However, considering that we may have more chance to encounter postsurgical OPS in the future with the development of complicated upper-airway surgery, we should be able to recognize and anticipate this complication. In our preoperative interview, we should specifically ask patients about their history of upper-airway surgery. If the patient has had surgery, an evaluation through detailed interview and close observation should be performed. We should also consult an otolaryngologist if we suspect a complication. Laryngofiberscopy will provide us with useful information for planning how to secure the patient's airway.

\section{Conclusions}

We reported a case of preoperatively undiagnosed OPS which resulted in unexpected difficult laryngoscope that necessitated a reschedule of surgery. Two factors prevented us from foreseeing the patient's OPS: insufficient communication due to language barrier and the patient's dismissal of dysphagia (her only problem due to OPS) as a harmless condition. We discussed the etiology of acquired OPS and we suggest that anesthesiologists be aware of this rare complication that could be on the rise.

This case was presented on September $5^{\text {th }}, 2015$, at the $55^{\text {th }}$ Annual Meeting of JSA Kanto-Tokyo Region.

\section{Consent to publish}

Written informed consent was obtained from the patient for publication of this case report and any accompanying images. A copy of the written consent is available for review by the editor-in-chief of this journal.

\section{Abbreviations}

OPS: oropharyngeal stenosis; OSA: obstructive sleep apnea; TORS: trans-oral robotic surgery; UPPP: uvulopalatopharyngoplasty.

\section{Competing interests \\ The authors declare that they have no competing interests.}

\section{Authors' contributions}

MN led the authors in preparing for this case report. HN evaluated the patient's OPS and wrote the corresponding part of the report, and made important input from the otolaryngologists' point of view. MM and $\mathrm{NI}$ evaluated the patient's airway in the OR and contributed to draft the manuscript. All authors read and approved the final manuscript.

\section{Author details}

${ }^{1}$ Department of Anesthesiology, Seibo International Catholic Hospital, 2-5-1, Nakaochiai, Shinjuku, Tokyo 161-8521, Japan. ${ }^{2}$ Department of Anesthesiology, Nihon University School of Medicine, 30-1, Oyaguchikamicho, Itabashi, Tokyo 173-8610, Japan. ${ }^{3}$ Department of Otolaryngology, Seibo International Catholic Hospital, 2-5-1, Nakaochiai, Shinjuku, Tokyo 161-8521, Japan.

Received: 10 September 2015 Accepted: 27 March 2016

Published online: 02 June 2016

References

1. Kheterpal S, Han R, Tremper KK, Shanks A, Tait AR, O'Reilly M, Ludwig TA. Incidence and predictors of difficult and impossible mask ventilation. Anesthesiology. 2006;105(5):885-91.

2. McLaughlin KE, Jacobs IN, Todd NW, Gussack GS, Carlson G. Management of nasopharyngeal and oropharyngeal stenosis in children. Laryngoscope. 1997;107:1322-31.

3. Stevenson EW. Cicatricial stenosis of the nasopharynx. A comprehensive review. Laryngoscope. 1969;79(12):2035-67.

4. Polychronopoulos VS, Prakash UBS. Airway involvement in sarcoidosis. Chest. 2009;136(5):1371-80.

5. Brooks GB. Pharyngeal stenosis in Behçet's syndrome. Arch Otolaryngol. 1983;109:338-40.

6. Verse T, Baisch A, Maurer JT, Stuck BA, Hörmann K. Multilevel surgery for obstructive sleep apnea: short-term results. Otolaryngol Head Neck Surg. 2006;134(4):571-7.

7. Li HY, Wang PC, Hsu CY, Chen NH, Lee LA, Fang TJ. Same-stage palatopharyngeal and hypopharyngeal surgery for severe obstructive sleep apnea. Acta Otolaryngol. 2004;124(7):820-6.

8. Prager JD, Hopkins BS, Propst EJ, Shott SR, Cotton RT. Oropharyngeal stenosis-a complication of multilevel, single-stage upper airway surgery in children. Arch Otolaryngol Head Neck Surg. 2010;136(11):1111-5.

9. Cohen DS, Sekhsaria V, McCormick ME, Lin HS. Circumferential oropharyngeal stenosis after single-stage transoral robotic surgery and standard uvulopalatopharyngoplasty for surgical treatment of obstructive sleep apnea: a report of five patients. Las Vegas: Trilogical Society Spring Meeting, Combined Otolaryngology Spring Meeting; 2014.

10. Oh Jl, Ham TI, Jeon SB, Kang MS, Shim HY. Unanticipated difficult endotracheal intubation due to an undiagnosed pharyngeal web. Korean J Anesthesiol. 2013;64(3):282-3. 